\title{
Polarimetry as a useful tool for the determination of binding constants between cyclodextrins and organic guest molecules
}

\author{
Paolo Lo Meo, * Francesca D’Anna, Serena Riela, \\ Michelangelo Gruttadauria and Renato Noto* \\ Dipartimento di Chimica Organica 'E. Paternò', Università degli Studi di Palermo, V.le delle Scienze, Parco d'Orleans II, \\ Pad. 17, 90128 Palermo, Italy
}

Received 20 September 2006; revised 10 October 2006; accepted 13 October 2006

Available online 3 November 2006

\begin{abstract}
Binding constants for cyclodextrin inclusion complexes can be easily estimated by means of simple polarimetric measurements. Determinations are as reliable and accurate as those obtained by means of other more sophisticated techniques, and take advantage by the limited waste of material required. Our results are briefly compared with literature values obtained by means of different techniques.

(c) 2006 Elsevier Ltd. All rights reserved.
\end{abstract}

An accurate evaluation of host-guest binding constants is a main issue in supramolecular chemistry. Indeed, the thermodynamics of the equilibrium process is an essential piece of information - together with structural information - in order to achieve a deep understanding of the binding phenomenon at a microscopic level, in particular when selective molecular recognition is implied. In order to estimate the binding constants between cyclodextrins (CDs, both native and chemically modified) and suitable organic guest molecules various methods have been developed and applied, exploiting different experimental techniques, which span from spectrometry (UV, fluorimetry, induced circular dichroism, NMR) to chromatography and electrophoresis, and to calorimetry. ${ }^{1}$ Although currently used methods show a tendency to become more and more sophisticated, this is not indeed a guarantee of accuracy or feasibility or valuability of the information obtained. In fact, estimation of binding constants for the same host-guest couple by means of different experimental methods can lead to strikingly different results, as shown by the various cases reported in the literature. ${ }^{2}$ In the present letter we intend to show how an old and simple technique such as polarimetry is able to provide an easy,

\footnotetext{
* Corresponding author. Tel.: +39 091596919; fax: +39 091596825; e-mail: rnoto@unipa.it
}

low-demanding (in terms of both time and materials) and accurate method for the evaluation of binding constants.

Any currently used measurement method suffers from its own drawbacks. Experienced researchers know them well and are able to evaluate how much the reliability of the results is affected by. In general, the detection of a variation upon binding in any suitable physical/chemical property $\boldsymbol{x}$ of the guest is exploited. This usually implies to work at a fixed guest concentration and varying the concentration $|\mathrm{CD}|$ of the $\mathrm{CD}$ host. The value of the binding constant $K$ is then obtained by regression analysis of the $\boldsymbol{x}$ versus $|\mathrm{CD}|$ data. Under this perspective, the use of spectrometric techniques has to cope with various problems. First, it is required that the maximum variation $\Delta \boldsymbol{x}_{0}$ of the observed property $\boldsymbol{x}$ upon inclusion (absorbance, NMR shift, fluorescence intensity, etc.) is large enough to be detected or estimated with sufficient precision, which is not always true or easy to achieve. For instance, UV is precluded to guests lacking a neat absorption band over $250 \mathrm{~nm}$; NMR induced shifts are sometimes small and may suffer for signal broadening; fluorimetry benefits a high sensitivity, but its application field is limited. Furthermore, provided that the $C D$ is generally used in large excess, the percent fraction of the complexed guest $\%{ }_{\mathrm{CDG}}$ is a hyperbolic function of $|\mathrm{CD}|$, according to relationship 1: 


$$
{ }^{0}{ }_{\mathrm{CDG}}=100 K|\mathrm{CD}|(1+K|\mathrm{CD}|)^{-1}
$$

Although there is not a general agreement about the particular point, our personal experience suggests that, in order to perform a reliable estimation of $K$, the ${ }^{\circ}{ }_{\mathrm{CDG}}$ value should never be less than $75 \%$ at the highest operational $\mathrm{CD}$ concentration. As a matter of fact, because $K$ and $\Delta x_{0}$ are determined at the same time as fitting parameters, their values (and the relevant indeterminations) are correlated, and consequently any error on the former will be reflected by an opposite error on the latter. This particular problem is shared by microcalorimetric measurements: in fact, the simultaneous determination of $\Delta H_{\mathrm{cplx}}^{\circ}$ and $K$ (which is the same as $\Delta G_{\text {cplx }}^{\circ}$ ) leads to the well-known 'enthalpy-entropy pseudo-compensation effect', which has been the object of intense debate and severe criticism until recently. ${ }^{3}$

From an operational point of view, spectrometric determinations require the preparation of a large number of sample solutions at various $|C D|$ values. On the grounds of the previous discussion, by means of simple algebraic passages we can easily obtain that $|\mathrm{CD}|$ should advisably increase at least up to $3 K^{-1}$ along the sample series. This implies a considerable waste of material, which is a serious problem when scarcely available (or difficult to synthesize) modified CDs have to be used. On the other hand, the highest $|\mathrm{CD}|$ allowable is anyhow limited by host solubility, which matter-of-factly imposes a lower limit to the $K$ values reliably measurable. The preparation of samples for fluorimetry is even more tedious, because very strict care is required to avoid spurious interferences. Determination of $K$ by HPLC (which exploits CDs as mobile phase additives, in particular for chiral selection purposes) ${ }^{4}$ is not more appealing. Also in this case large amounts of materials are required; moreover, analyses are lengthy and a strict control of experimental conditions is required to have good data reproducibility.

Curiously, simple polarimetry has not attracted the attention of researchers as a tool for binding constants evaluation. At the best of our knowledge only one occasional report ${ }^{5}$ can be found in the recent literature. However, we think that polarimetry deserves much more consideration, as it can be profitably used to get both thermodynamic and structural information. Our approach exploits the characteristics of the host rather than the guest, and is based on a few simple ideas. The optical activity of CDs is due not only to the presence of a given number of chiral glucose units in their structure, but it is also strongly affected by their mutual spatial arrangement, as well as by the overall conformational behavior of the whole macrocycle. ${ }^{6}$ In fact, the usual representation of CDs as $C_{n}$ symmetric buckets is only a mere pictorial artifact accounting for their mean dynamic behavior. Their real structures undergo a significant degree of de-symmetrization, ${ }^{7}$ because of the mutual torsional displacement of the glucose units. Moreover, helicity character has been attributed by to both the hydroxyl rims of the CD macrocycle, in order to explain the chiral recognition properties of some modified CDs. ${ }^{8}$ It is noteworthy that the molar optical activities ${ }^{9}\left[\Theta_{\mathrm{CD}}\right]_{\mathrm{D}}^{25}$ of native CDs $(+140 \pm 1,+180 \pm 1$ and $+224 \pm 1 \mathrm{deg} \mathrm{dm}^{-1} \mathrm{M}^{-1}$ for $\alpha \mathrm{CD}, \beta \mathrm{CD}$ and $\gamma \mathrm{CD}$, respectively, in water ${ }^{10}$ are significantly different from the sum of the contributions due to the single $\alpha$-glucose units. Thus, we can reasonably expect to observe a variation of the optical activity of the CD upon inclusion of a guest, because of both the conformational changes of the macrocycle, and the local dipolar micro-environment effect of the guest itself.

The estimation of $K$ values by means of polarimetry requires working at a fixed $\mathrm{CD}$ concentration and variable concentration of the guest. In order to perform a reliable determination only few requirements should be satisfied. The CD concentration $|C D|$ should be sufficiently high to allow a reliable measurement of the optical rotation of the sample. Using a modern routinary equipment, a detection sensitivity of $0.001 \mathrm{deg}$ or less can be easily achieved; consequently, considered the molar optical activities of native cyclodextrins, the host concentration may be reduced down to $5 \times 10^{-4} \mathrm{M}^{11}$ Moreover, the solubility of the guest in the chosen solvent medium must be comparable or higher than the operational $|C D|$ value. This is necessary to ensure that a significant fraction of the $\mathrm{CD}$ is engaged in complex formation at equilibrium, advisably $75 \%$ at least for the sample at the highest guest concentration. Under this condition, by means of simple algebraic passages we can easily derive the following numerical condition 2 to the solubility $|\mathrm{G}|_{0}$ of the guest:

$$
|G|_{0} \geqslant 3 K^{-1}+0.75|\mathrm{CD}|
$$

from which we can also deduce that guest solubility imposes a downward limitation to the $K$ values reliably estimable, according to relationship 3 :

$$
K \geqslant\left(|G|_{0} / 3+|\mathrm{CD}| / 4\right)^{-1}
$$

The experimental procedure developed by us provides the preparation of a series of sample solutions by mixing variable micro-volumes of a concentrated methanol guest solution (dispensed by means of a high precision microsyringe) to fixed amounts of a unique mother solution of the CD in a suitable aqueous buffer. It is worth noting that this procedure is particularly advantageous, because sample preparation is fast and optimization of materials used can be easily achieved, which allows wastes minimization. Provided that the amount of methanol guest solution added to each sample is small with respect to the total volume, we can assume that samples volumes follow the linear relationship 4:

$$
V_{i}=V_{0}\left(1+f v_{i} / V_{0}\right)
$$

where $V_{0}$ is the fixed volume of the CD solution, $v_{i}$ the variable volume of guest solution added to each sample, and the coefficient $f$ matter-of-factly accounts for the partial molar volumes of methanol in water. ${ }^{12}$ Moreover, we can also reasonably assume that the position of the binding equilibrium is not affected to a significant extent, that is, the effect of the methanol amount on $K$ is negligible with respect to experimental indeterminations. Then, considered a light path of $1 \mathrm{dm}$, the optical rotation $\vartheta_{i}$ for each sample can be expressed as 5: 


$$
\begin{aligned}
\vartheta_{i} & =\left[\Theta_{\mathrm{CD}}\right]|\mathrm{CD}|+\left[\Theta_{\mathrm{CDG}}\right]|\mathrm{CDG}| \\
& =\vartheta_{0}+[\Delta \Theta]|\mathrm{CDG}|
\end{aligned}
$$

where $|\mathrm{CD}|$ and $|\mathrm{CDG}|$ are the equilibrium concentrations of the $\mathrm{CD}$ and the complex, respectively $\left[\Theta_{\mathrm{CD}}\right]$, and $\left[\Theta_{\mathrm{CDG}}\right]$ are the relevant molar optical activities, $\vartheta_{0}$ is the optical rotation of the sample without guest, and $[\Delta \Theta]=\left[\Theta_{\mathrm{CDG}}\right]-\left[\Theta_{\mathrm{CD}}\right]$. Because host and guest are present in the samples in comparable amounts, application of the usual Benesi-Hildebrand treatment to experimental data is unsuitable. The correct expression 6 for $|\mathrm{CDG}|$ must be rather deduced analytically. The latter one can be used in turn for data processing, to obtain the values of both $[\Delta \Theta]$ and $K$ :

$$
\begin{aligned}
\vartheta_{i}= & \frac{\vartheta_{0}+\frac{[\Delta \Theta]}{2}}{1+f v_{i} / V_{0}}\left(\mathrm{CD}_{0}^{0}+G_{0}^{0} \frac{v_{i}}{V_{0}}+\frac{1+f v_{i} / V_{0}}{K}\right. \\
& \left.-\sqrt{\left(\mathrm{CD}_{0}^{0}+G_{0}^{0} \frac{v_{i}}{V_{0}}+\frac{1+f v_{i} / V_{0}}{K}\right)^{2}-4 C D_{0}^{0} G_{0}^{0} \frac{v_{i}}{V_{0}}}\right)
\end{aligned}
$$

where $\mathrm{CD}_{0}$ and $G_{0}$ are the concentrations for the mother solutions of the $\mathrm{CD}$ and the guest, respectively. ${ }^{13} \mathrm{~A}$ typical fitting example is reported in Figure 1.

In order to explore the application of polarimetry to $K$ measurement, we investigated the interaction at $25 \pm 1{ }^{\circ} \mathrm{C}$ between native cyclodextrins $\alpha \mathrm{CD}$ or $\beta C D$ and model organic guests $\mathbf{1}-\mathbf{6}$ (Fig. 2) in suitable aqueous buffer solution at various $\mathrm{pH}$ values $(2.5,6.0,9.2$, $\left.11.0, I=10^{-1} \mathrm{M}\right)$. Guests $1-6$ were chosen in order to show significant variations in molecular properties such as size, dipole momentum, and electric charge as a function of the aqueous solvent buffer. For all these guests but one (2) relevant $K$ values had been already obtained with other methodologies and reported (Table 1).

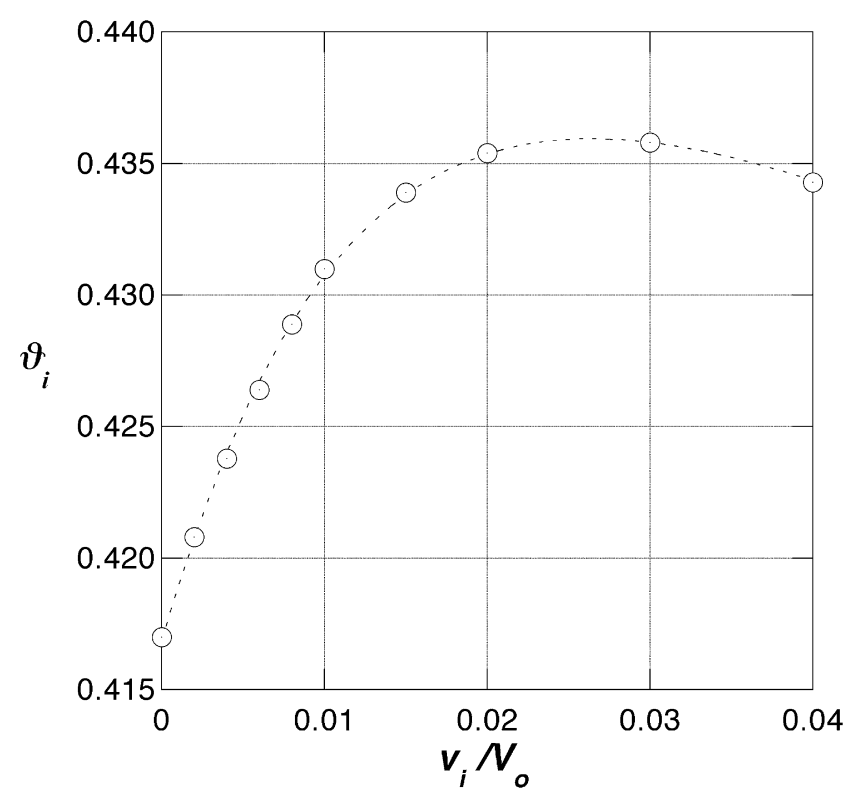

Figure 1.<smiles>OC1CCCCC1</smiles><smiles>C1CN2CCN1CC2</smiles><smiles>O=C(O)C12CC3CC(CC(C3)C1)C2</smiles><smiles>O=C(O)c1ccccc1</smiles>

1

2

3

4

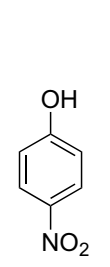

5

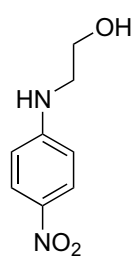

6

Figure 2.

Table 1.

\begin{tabular}{llrrl}
\hline Host & Guest & \multicolumn{1}{c}{$\mathrm{pH}$} & \multicolumn{1}{c}{$[\Delta \Theta]^{\mathrm{a}}$} & $\log K^{\mathrm{a}, \mathrm{b}}$ \\
\hline$\alpha \mathrm{CD}$ & $\mathbf{4}$ & 2.5 & $+12.7 \pm 0.3$ & $3.13 \pm 0.05^{\mathrm{c}}$ \\
& $\mathbf{5}$ & 2.5 & $+25.9 \pm 0.3$ & $2.52 \pm 0.03^{\mathrm{d}}$ \\
& $\mathbf{5}$ & 9.2 & $+104.2 \pm 1.8$ & $3.52 \pm 0.06^{\mathrm{e}}$ \\
& $\mathbf{6}$ & 6.0 & $+90.2 \pm 1.6$ & $3.19 \pm 0.04^{\mathrm{f}}$ \\
$\beta \mathrm{CD}$ & $\mathbf{1}$ & 6.0 & $-11.5 \pm 0.3$ & $2.82 \pm 0.04^{\mathrm{g}}$ \\
& $\mathbf{2}$ & 11.0 & $-12.7 \pm 0.4$ & $2.29 \pm 0.05$ \\
& $\mathbf{3}$ & 9.2 & $-21.9 \pm 0.3$ & $4.09 \pm 0.07^{\mathrm{h}}$ \\
& $\mathbf{4}$ & 2.5 & $+9.8 \pm 0.3$ & $2.98 \pm 0.06^{\mathrm{i}}$ \\
& $\mathbf{5}$ & 2.5 & $+13.5 \pm 0.2$ & $2.87 \pm 0.04^{\mathrm{j}}$ \\
& $\mathbf{5}$ & 9.2 & $+81.7 \pm 0.2$ & $2.91 \pm 0.01^{\mathrm{k}}$ \\
& $\mathbf{6}$ & 6.0 & $+74.6 \pm 0.8$ & $2.78 \pm 0.01^{1}$ \\
\hline
\end{tabular}

${ }^{\mathrm{a}}$ Mean values on at least two independent determinations.

${ }^{\mathrm{b}}$ Literature data are from Refs. 2 and 3; pot: potentiometry, cal: calorimetry, cd: circular dichroism, uv: spectrophotometry, lc: liquid chromatography.

${ }^{\mathrm{c}}$ Lit. 2.88 (pot); 3.0 (cal); 2.52 (cd).

${ }^{\mathrm{d}}$ Lit. various values in the range $2.01-2.59$ (cal, uv, cd).

${ }^{\mathrm{e}}$ Lit. various values in the range 3.09-3.55 (cal, uv, $\mathrm{pH} 9.5-11.1$ ).

${ }^{\mathrm{f}}$ Lit. 3.02 (uv).

${ }^{\mathrm{g}}$ Lit. 2.85 (pH 6.9, cal), 2.70 (uv).

${ }^{\text {h }}$ Lit. 4.51 (pH 7.2, cal); 4.60 (pH 7.0, cal); 4.29 (pH 8.5, cal).

${ }^{\text {i }}$ Lit. 2.1 (cal); 3.26 (cal); 2.74 (pot); 2.52 (cd).

${ }^{\mathrm{j}}$ Lit. various values in the range $2.41-2.54$ (cal, uv); 2.28 (cd); 1.75 (pH $3.57,1 \mathrm{c})$.

${ }^{\mathrm{k}}$ Lit. 3.25 (pH 9.0, cal); 2.56 (pH 9.5, cal); 2.97 (pH 10.0, uv); 2.80 (pH 11.0, uv); 2.76 (pH 11.1, cal).

${ }^{1}$ Lit. 2.78 (uv).

Our measurement method showed in general a satisfactory data reproducibility. In particular, with guest $\mathbf{5}$ highly reproducible results were obtained also drastically changing the concentrations of the mother solutions. The $K$ values found are generally in good to excellent agreement with literature reports, accounting for the overall reliability of the method. The values of $K$ reported herein range from $195(2 \cdot \beta C D)$ up to $12300 \mathrm{M}^{-1}$ (3· $\left.\beta \mathrm{CD}\right)$; however, our experience suggests us that reliable estimations might be easily performed out of this range too. Noticeably, we were able to perform the otherwise difficult determination of $K$ for the couple $2 \cdot \beta C D$.

A satisfactory agreement of $K$ data with literature reports is found for guests $\mathbf{1}, \mathbf{3}$ and in particular $\mathbf{6}$. Guests 4 (anion) and $\mathbf{5}$ (both free acid and anionic forms at $\mathrm{pH} 2.5$ and 9.2, respectively) need an articulated discussion because of the different $K$ values provided by literature (for instance, from 126 up to 1820 for $4 \cdot \beta C D$, depending on the experimental method). In four cases among six, our datum lies within the range of literature 
reports (best agreement is usually found with UV data). In two cases only $(\mathbf{4} \cdot \alpha \mathrm{CD}, \mathbf{5} \cdot \beta \mathrm{CD}$ at $\mathrm{pH} 2.5)$ our estimation is significantly higher than literature reports. Regarding $[\Delta \Theta]$ values, our data are not sufficient for a systematic study; however, they account for a remarkable difference in behavior between aliphatic (1-3) and aromatic (4-6) guests, and seem easily rationalizable in terms of both guest bulk and dipolar effects.

As a final remark, it is interesting to stress that the polarimetric method proposed here allows to compare under homogeneous conditions guests very different from a structural point of view, regardless of their actual properties. By contrast, methods based on the detection of guest properties, limit the possibility to perform homogeneous comparisons to the class of guests sensitive to the same technique.

\section{Acknowledgments}

Italian MIUR (National Research Project 'Non-aromatic heterocycles in stereocontrolled processes') and University of Palermo are acknowledged for financial support.

\section{References and notes}

1. Connors, K. A. In Comprehensive Supramolecular Chemistry; Szejtli, J., Osa, T., Eds.; Pergamon: New York, 1996; Vol. 3, pp 205-240.

2. Rekharsky, M.; Inoue, Y. Chem. Rev. 1998, 98, 18751917.
3. Lo Meo, P.; D’Anna, F.; Gruttadauria, M.; Riela, S.; Noto, R. Tetrahedron 2004, 60, 9099-9111, and references therein.

4. Snopek, J.; Smolková-Keulemansová, E.; Cserháti, T.; Gham, K. H.; Stallcup, A. In Comprehensive Supramolecular Chemistry; Szejtli, J., Osa, T., Eds.; Pergamon: New York, 1996; Vol. 3, pp 515-571.

5. Bergeron, R. J.; Almy Channing, M.; Gibeily, G. J.; Pillor, D. M. J. Am. Chem. Soc. 1977, 99, 5146-5151.

6. Rees, D. A. J. Chem. Soc. (B) 1970, 877-884.

7. Lipkowitz, K. B. Chem. Rev. 1998, 98, 1829-1873.

8. Kano, K.; Hasegawa, H. J. Am. Chem. Soc. 2001, 123, 10616-10627, and references therein.

9. Despite usual practice, we prefer to abandon the common use of specific optical activities, replacing them with the molar optical activities $[\Theta]_{\mathrm{D}}^{t^{\circ} \mathrm{C}}$, defined as the optical rotation observed for an ideal $1 \mathrm{M}$ solution (under all the other usual conditions). This choice in our opinion simplifies the mathematical treatment and allows a better data comparison.

10. From data reported in Ref. 6 slightly different values can be calculated, probably because of the different temperature.

11. The procedure reported in Ref. 5 provides a CD concentration $1.6 \times 10^{-4} \mathrm{M}$. In our opinion such low concentrations should be avoided, because the experimental indetermination on $\vartheta_{i}$ values would be comparable with the variations in optical rotations.

12. The value 0.938 was estimated for $f$ on the grounds of density data for methanol-water mixtures reported in CRC Handbook of Chemistry and Physics, 80th ed.; Lide, D. R., Ed.; CRC Press: New York, 1999; pp 8-69.

13. According to the algebraic properties of Eq. 6, it is useful to remark that, when $\Delta \Theta$ is positive, $\vartheta_{i}$ is an increasing function of $v_{i} / V_{0}$; however, it may also pass through a maximum and then decrease if, at the same time, both $K$ and $G_{0}$ are large. Differently, if $\Delta \Theta$ is negative, $\vartheta_{i}$ is a monotonic decreasing function of $v_{i} / V_{0}$. 